\title{
An iliopsoas abscess caused by Parvimonas micra: a case report
}

\author{
Toyomitsu Sawai $^{1 *}$, Satoru Koga', Shotaro Ide ${ }^{1}$, Sumako Yoshioka', Nobuko Matsuo ${ }^{1}$ and Hiroshi Mukae ${ }^{2}$
}

\begin{abstract}
Background: Parvimonas micra, a Gram-positive anaerobic coccus, is a rare pathogen for psoas abscess. We describe a case of a patient with iliopsoas abscess caused by $P$. micra.

Case presentation: An 81-year-old Asian man presented to our department with complaints of fever since the preceding day. Abdominal computed tomography revealed the presence of a low-density mass in the right iliopsoas muscle indicative of a psoas abscess. Computed tomography-guided percutaneous drainage of the psoas abscess was performed. Results of organism cultures of the abscess and blood were positive for P. micra. However, our patient had no known primary focus of infection. On the basis of these findings, a primary psoas abscess caused by $P$. micra was diagnosed, and treatment with ampicillin/sulbactam $1.5 \mathrm{~g}$, administered intravenously every $8 \mathrm{~h}$, was initiated. By day 7 , the patient's white blood cell count normalized. By day 20, his C-reactive protein level was decreased to $0.35 \mathrm{mg} / \mathrm{dl}$.

Conclusion: lliopsoas abscesses caused by anaerobic bacteria are relatively rare, and iliopsoas abscesses caused by $P$. micra are especially rare. Our patient's case revealed that $P$. micra can cause iliopsoas abscess. Therefore, clinicians should be aware of the possibility that $P$. micra may cause iliopsoas abscess.
\end{abstract}

Keywords: Primary iliopsoas abscess, Parvimonas micra, CT-guided percutaneous drainage, Ampicillin/sulbactam

\section{Introduction}

Parvimonas micra, formerly known as Peptostreptococcus micros and Micromonas micra, is a fastidious, anaerobic, Gram-positive coccus that is normally found in the human dental and gastrointestinal flora [1]. P. micra is related to polymicrobial infections, especially in the oral cavity. It has also been implicated in more invasive infections, such as vertebral osteomyelitis, spondylodiscitis, arthritis, endocarditis, and pleuritis [2-5]. Although a variety of infections associated with $P$. micra have been reported, psoas abscesses caused by this organism have been described only rarely. We report a case of a patient with iliopsoas abscess caused by P. micra.

\section{Case presentation}

An 81-year-old Asian man presented to our department complaining of fever since the preceding day. The patient had been under treatment for the previous 3 years for chronic heart failure and chronic renal failure. He

\footnotetext{
* Correspondence: toyosawai@yahoo.co.jp

${ }^{1}$ Department of Respiratory Medicine, Nagasaki Harbor Medical Center, 6-39

Shinchi-machi, Nagasaki 850-8555, Japan

Full list of author information is available at the end of the article
}

did not have a history of malignancy, diabetes mellitus, cytotoxic therapy, or corticosteroid use, and no foreign bodies had been implanted. The patient's family history was unremarkable. Physical examination revealed a heart rate of 101 beats/min, blood pressure of $87 / 48 \mathrm{mmHg}$, respiratory rate of 20 breaths $/ \mathrm{min}$, temperature of $37.0^{\circ}$ $\mathrm{C}$, and oxygen saturation of $87 \%$ on room air. He had no caries or periodontitis. Results of respiratory, cardiac, and abdominal examinations were unremarkable. Limb examination demonstrated mild edema of both legs. Abdominal computed tomography (CT) showed a lowdensity mass in the right iliopsoas muscle indicative of an iliopsoas abscess (Fig. 1). The patient's white blood cell count, C-reactive protein (CRP), and procalcitonin levels were $19,400 / \mu \mathrm{l}, 13.35 \mathrm{mg} / \mathrm{dl}$, and $3.950 \mathrm{ng} / \mathrm{ml}$, respectively. Serum blood urea nitrogen and creatinine were elevated at $77.2 \mathrm{mg} / \mathrm{dl}$ and $3.69 \mathrm{mg} / \mathrm{dl}$, respectively. A CT-guided percutaneous drainage of the psoas abscess was performed, and an indwelling catheter was placed. Gram staining of the drained fluid revealed many neutrophils and Gram-positive streptococci. On the basis of these findings, a presumptive diagnosis of iliopsoas abscess caused by Streptococcus species was made, and 


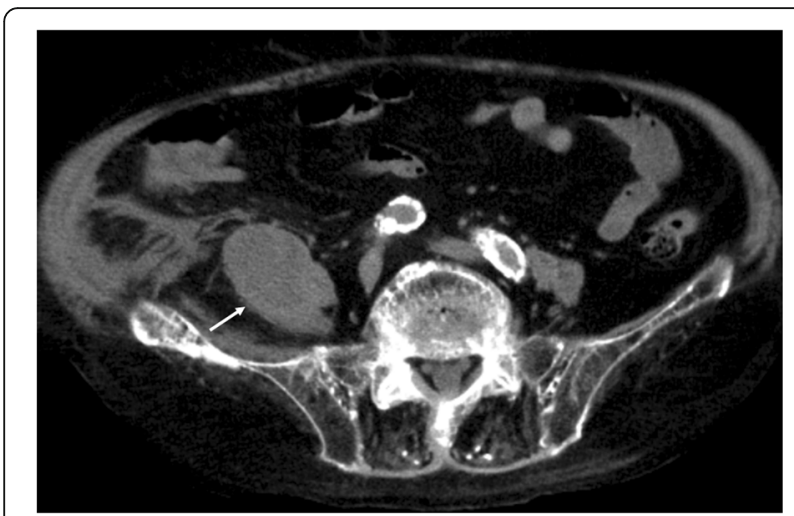

Fig. 1 Abdominal computed tomography on admission showed a low-density mass in the right iliopsoas muscle (white arrow)

treatment with ampicillin/sulbactam (ABPC/SBT) $1.5 \mathrm{~g}$, administered intravenously every $8 \mathrm{~h}$, was initiated. Results of organism cultures of the abscess and blood were positive, and P. micra was identified by using the API ZYM system (Sysmex-bioMérieux Co. Ltd., Tokyo, Japan), with the organism exhibiting susceptibility to penicillin G, ampicillin, clindamycin, and meropenem. By day 7, the patient's white blood cell count normalized. By day 20, his CRP level was decreased to $0.35 \mathrm{mg} /$ $\mathrm{dl}$. Therefore, the pigtail catheter was removed. The patient died of peritonitis due to colon diverticulum perforation after 5 weeks of treatment. An autopsy revealed no right iliopsoas abscess at the time of death.

\section{Discussion}

Iliopsoas abscess is a rare life-threatening infection with a varied symptomology and etiology. The diagnosis and effective management are frequently delayed because of the nonspecific nature of clinical symptoms. Primary and secondary iliopsoas abscesses are most frequently caused by Staphylococcus aureus and Escherichia coli, respectively [6, 7]. A review of the literature showed that there have been very few cases of iliopsoas abscesses caused by $P$. micra $[7,8]$. Our patient's case had no known primary focus of infection. Given that the iliopsoas abscess in our patient apparently arose as a result of hematogenous dissemination from an unknown distant focus of infection, a diagnosis of primary iliopsoas abscess was made. Navarro Lopez et al. reported that Peptostreptococcus species were isolated from 5 of $72(6.9 \%)$ patients with secondary iliopsoas abscess, whereas this organism was isolated from 1 of 21 (4.8\%) patients with primary iliopsoas abscess [8]. Wada et al. reported a case of primary $P$. micra iliopsoas abscess due to hematogenous dissemination from a central venous catheter [9]. However, Wada et al. did not collect samples from the abscess, and P. micra iliopsoas abscess was diagnosed solely on the basis of isolation from the patient's blood culture. In a review of 31 case reports with infections caused by P. micra, Cobo et al. reported that the infection site was the vertebral spine in 14 (45.1\%) cases; joints and heart valves in $5(16.1 \%)$ cases each, pleura in $3(9.6 \%)$ cases; and in brain, lung and head and neck, chest wall, or meninges in $1(3.2 \%)$ case each. A case of iliopsoas abscess had not previously been reported [5]; accordingly, our patient's case appears to be a definitive case of a primary iliopsoas abscess caused by $P$. micra.

Iliopsoas abscess remains a relatively uncommon clinical entity. Since Mynter's original description of pyogenic iliopsoas abscess in 1881 [10], more than 500 cases have been reported in the world literature $[8,11$, 12]. The classic triad of back pain, limp, and fever may be present, but subsequent case studies have suggested that this triad may be detected in only $30 \%$ of cases [13]. Indeed, in our patient, the only clinical symptom was fever. Many patients with psoas abscess will present with nonspecific features such as malaise and low-grade pyrexia, meaning that there frequently may be delays in diagnosis. Accordingly, CT has become the standard means of diagnosing psoas abscess, with a sensitivity of over $90 \%$ and specificity of over $80 \%$ [14]. Although magnetic resonance imaging (MRI) has been shown to be more sensitive than $\mathrm{CT}$ in detecting intra-abdominal abscess [15], MRI is complex, and radiologists are not freely able to report on MRI. An additional advantage is that CT guidance allows safe percutaneous needle placement and the collection of samples from the psoas abscess. Our patient was diagnosed by using abdominal CT, and CT-guided percutaneous drainage was performed.

Blood cultures and direct abscess aspirate are the most definitive diagnostic tools. However, a definitive microbiological diagnosis for psoas abscess has been reported in only $75 \%$ of cases. Specifically, the rates of diagnosis by infected fluid and blood culture have been reported to be $74.3 \%$ and $31.5 \%$, respectively [8]. S. aureus and $E$. coli are the most common organisms detected in primary and secondary iliopsoas abscess, respectively. Iliopsoas abscesses caused by anaerobic bacteria are relatively rare, and iliopsoas abscesses caused by $P$. micra appear to be especially rare. The anaerobe that most often causes anaerobic iliopsoas abscess is Bacteroides species [8]. However, P. micra might be an underreported causative pathogen in iliopsoas abscesses, because the organism is difficult to culture and identify. Cobo et al. reported that $P$. micra infections were diagnosed on the basis of infected fluids in $38.7 \%$ of cases, blood cultures in $29 \%$, and molecular techniques in $22.5 \%$ [5]. In recent times, as new methods (e.g., $16 \mathrm{~S}$ ribosomal RNA sequencing [3], matrix-assisted laser desorption ionization time-of-flight mass spectrometry [4], and melting temperature mapping [16]) have become more available 
in routine diagnostic laboratories, $P$. micra has been increasingly detected in various invasive human infections.

Although the first-line treatment in the literature is broad-spectrum antibiotic that will cover S. aureus and also any other possible primary source for the iliopsoas abscess, some authors have suggested that targeted antibiotics may be sufficient to treat abscesses up to $60 \mathrm{~mm}$ in width [17]. Additionally, it has since been established that image-guided percutaneous drainage is very effective and safe [18]. Post-drainage antibiotic therapy should be tailored to the organism isolated. The treatment for iliopsoas abscess caused by P. micra includes antimicrobial therapy with or without drainage. However, a standard antibiotic therapy for $P$. micra infections has not yet been established. P. micra is usually susceptible to antibiotics, including penicillin, imipenem, clindamycin, and metronidazole, although metronidazole-resistant strains of $P$. micra have been reported [19-21]. In general, metronidazole should not be administered as empiric therapy until susceptibility testing results are available. Accordingly, we selected intravenous ABPC/SBT antibiotic therapy with drainage of the abscess, a regimen that successfully treated the abscess in our patient. However, the optimal duration of therapy for iliopsoas abscess is unknown.

\section{Conclusions}

Iliopsoas abscesses caused by anaerobic bacteria are relatively rare, and iliopsoas abscesses caused by $P$. micra appear to be especially rare. Our patient's case revealed that $P$. micra can cause primary iliopsoas abscess. Therefore, clinicians should be aware of the possibility that $P$. micra may cause primary iliopsoas abscess.

\section{Abbreviations}

ABPC/SBT: Ampicillin/sulbactam; CRP: C-reactive protein; CT: Computed tomography; MRI: Magnetic resonance imaging

\section{Acknowledgements}

The authors wish to acknowledge the patient.

\section{Funding}

None.

\section{Availability of data and materials}

All available data is presented in the main paper

\section{Authors' contributions}

TS managed the patient and reviewed the literature. SK and SI contributed to the collection of patient data. SY analyzed the radiologic findings. NM performed microbiological tests. TS was the main writer of the manuscript. HM moderated the manuscript. All authors read and approved the final manuscript.

\section{Ethics approval and consent to participate}

Not applicable.

\section{Consent for publication}

Written informed consent was obtained from the patient's next of kin for publication of this case report and any accompanying images. A copy of the written consent is available for review by the Editor-in-Chief of this journal.

\section{Competing interests}

The authors declare that they have no competing interests.

\section{Publisher's Note}

Springer Nature remains neutral with regard to jurisdictional claims in published maps and institutional affiliations.

\section{Author details}

'Department of Respiratory Medicine, Nagasaki Harbor Medical Center, 6-39 Shinchi-machi, Nagasaki 850-8555, Japan. ${ }^{2}$ Second Department of Internal Medicine, Nagasaki University Hospital, 1-7-1 Sakamoto-machi, Nagasaki, Japan.

Received: 20 February 2018 Accepted: 1 February 2019

Published online: 01 March 2019

\section{References}

1. Tindall BJ, Euzeby JP. Proposal of Parvimonas gen. nov. and Quatrionicoccus gen. nov. as replacements for the illegitimate, prokaryotic, generic names Micromonas Murdoch and Shah 2000 and Quadricoccus Maszenan et al. 2002, respectively. Int J Syst Evol Microbioliagn Microbiol. 2006;56:2711-3.

2. Murdoch DA. Gram-positive anaerobic cocci. Clin Microbiol Rev. 1998;11:81-120

3. Dietvorst M, Roerdink R, Leenders ACAP, Kiel MA, Bom LPA. Acute monoarthritis of the knee: A case report of infection with Parvimonas micra and concomitant pseudogout. J Bone Joint Infect. 2016;1:65-7.

4. Cleaver LM, Palanivel S, Mack D, Warren S. A case of polymicrobial anaerobic spondylodiscitis due to Parvimonas micra and Fusobacterium nucleatum. JMM Case Rep. 2017:4:e005092.

5. Cobo F, Rodriguez-Granger J, Sampedro A, Aliaga-Martinez L, Navarro-Mari JM. Pleural effusion due to Parvimonas micra: a case report and a literature review of 30 cases. Rev Esp Quimioter. 2017:30:285-92.

6. Desandre AR, Cottone FJ, Evers ML. Iliopsoas abscess: etiology, diagnosis, and treatment. Am Surg. 1995;61:1089-91.

7. Shields D, Robinson P, Crowley TP. Iliopsoas abscess: a review and update on the literature. Int J Surg. 2012:10:466-9.

8. Navarro Lopez V, Ramos JM, Meseguer V, Perez Arellano JL, Serrano R, Garcia Ordonez MA, Peralta G, Boix V, Pardo J, Conde A, Salgado F, Gutierrez F, GTI-SEMI Group. Microbiology and outcome of iliopsoas abscess in 124 patients. Medicine (Baltimore). 2009:88:120-30.

9. Wada Y, Sato M, Saito A, Gejyo F. Infection-associated hemophagocytic syndrome in a diabetic patient undergoing chronic hemodialysis. Clin Exp Nephrol. 2003;7:163-6.

10. Mynter H. Acute psoitis. Buffalo Med Surg J. 1881;21:202.

11. Ricci MA, Rose FB, Meyer KK. Pyogenic psoas abscess: world-wide variations in etiology. World J Surg. 1986;10:834-43.

12. Gruenwald I, Abrahamson J, Cohen O. Psoas abscess: case report and review of the literature. J Urol. 1992;147:1624-6.

13. Chern CH, Hu SC, Kao WF, Tsai J, Yen D, Lee CH. Psoas abscess: making an early diagnosis in the ED. Am J Emerg Med. 1997;15:83-8.

14. MacGillivray DC, Valentine RJ, Johnson JA. Strategies in the management of pyogenic psoas abscesses. Am J Surg. 1991;57:701-5.

15. Wall SD, Fisher MR, Amparo EG, Hricak H, Higgins CB. Magnetic resonance imaging in the evaluation of abscesses. AJR Am J Roentgenol. 1985;144:1217-21.

16. Higashi $Y$, Nakamura S, Niimi H, Ueno T, Matsumoto K, Kawago K, Sakamaki I, Kitajima I, Yamamoto Y. Spondylodiscitis due to Parvimonas micra diagnosed by the melting temperature mapping method: a case report. BMC Infect Dis. 2017;17:584.

17. Yacoub W, Sohn H, Chan S, Petrosyan M. Psoas abscess rarely requires surgical intervention. Am J Surg. 2008:196:223-7.

18. Dinc H, Ahmetoglu A, Baykal S, Sari A, Sayil O, Gumele HR. Image guided percutaneous of tuberculous iliopsoas and spondylodiskitic abscesses: midterm results. Radiology. 2002;225:353-8.

19. Ubeda P, Todoli J, Saro E, Gomez MD, Perez Belles C, Gobernado MB. Subacute infectious endocarditis in prosthetic valve by Peptostreptococcus micros [in Spanish]. Enferm Infecc Microbiol Clin. 1998;16:63-4.

20. Veloo AC, Welling GW, Degener JE. Antimicrobial susceptibility of clinicallyrelevant Gram-positive anaerobic cocci collected over a three-year period in the Netherlands. Antimicrob Agents Chemother. 2011;55:1199-203.

21. Jones SL, Riordan JW, Glasgow AL, Botes J, Boutlis CS. Two cases of spondylodiscitis caused by Parvimonas micra. Intern Med J. 2015:45:1090-1. 